\title{
openheart Uncovering the treatable burden of severe aortic stenosis in the UK
}

\author{
Geoffrey A Strange (1) , ${ }^{1,2}$ Simon Stewart (1) , ${ }^{3,4}$ Nick Curzen (1) , Simon Ray (1) ,6 \\ Simon Kendall, ${ }^{7}$ Peter Braidley, ${ }^{8}$ Keith Pearce, ${ }^{9}$ Renzo Pessotto, ${ }^{10}$ \\ David Playford (D) , ${ }^{1}$ Huon H Gray ${ }^{11}$
}

\begin{abstract}
- Additional supplemental material is published online only. To view, please visit the journal online (http://dx.doi.org/10.
\end{abstract} 1136/openhrt-2021-001783).

To cite: Strange GA, Stewart S, Curzen $\mathrm{N}$, et al. Uncovering the treatable burden of severe aortic stenosis in the UK. Open Heart 2022;9:e001783. doi:10.1136/ openhrt-2021-001783

Received 13 July 2021 Accepted 1 November 2021
Check for updates

(c) Author(s) (or their employer(s)) 2022. Re-use permitted under CC BY-NC. No commercial re-use. See rights and permissions. Published by BMJ.

For numbered affiliations see end of article.

Correspondence to Dr Geoffrey A Strange; gstrange@neda.net.au

\section{ABSTRACT}

Objective To estimate the population prevalence and treatable burden of severe aortic stenosis (AS) in the UK. Methods We adapted a contemporary model of the population profile of symptomatic and asymptomatic severe AS in Europe and North America to estimate the number of people aged $\geq 55$ years in the UK who might benefit from surgical aortic valve replacement (SAVR) or transcatheter aortic valve implantation (TAVI).

Results With a point prevalence of $1.48 \%$, we estimate that 291448 men and women aged $\geq 55$ years in the UK had severe AS in 2019. Of these, $68.3 \%(199059,95 \% \mathrm{Cl}$ 177201 to 221355 people) would have been symptomatic and, therefore, more readily treated according to their surgical risk profile; the remaining $31.7 \%$ of cases (92389, 95\% Cl 70093 to 144247) being asymptomatic. Based on historical patterns of intervention, $58.4 \%$ (116251, 95\% Cl 106895 to 125606$)$ of the 199059 symptomatic cases would qualify for SAVR, with 7208 $(95 \% \mathrm{Cl} 7091$ to 7234$)$ being assessed as being in a high, preoperative surgical risk category. Among the remaining $41.6 \%(82809,95 \% \mathrm{Cl} 73453$ to 92164$)$ of cases potentially unsuitable for SAVR, an estimated $61.7 \%$ (51 093, 95\% Cl 34780 to 67655 ) might be suitable for TAVI. We estimate that 172859 out of 291448 prevalent cases of severe AS (59.3\%) will subsequently die within 5 years without proactive management.

Conclusions These data suggest a high burden of severe AS in the UK requiring surgical or transcatheter intervention that challenges the ongoing capacity of the National Health Service to meet the needs of those affected.

\section{INTRODUCTION}

Aortic stenosis (AS) is one of the most common acquired forms of heart valve disease requiring clinical intervention. ${ }^{12}$ As highlighted by the OxVALVE Study, ${ }^{3}$ a significant portion of the UK population $(1.3 \%$ of their cohort of individuals age $\geq 65$ years had undiagnosed AS) will remain undetected during their lifetime or experience a 'late' diagnosis when their condition reaches an advanced, symptomatic stage. ${ }^{4}$ The prevalence of AS correlates strongly with advancing age and is a major cause of death among older individuals. ${ }^{12}$ For example, within the large National

\section{Key questions}

What is already known about this subject?

- Severe aortic stenosis (AS) has a high mortality if left untreated.

- The number of individuals who might benefit from treatment in the UK is uncertain, although anticipated to be large.

What does this study add?

- This study provides robust estimates of the overall burden of severe AS across the UK and the indicative number of individuals who would benefit from surgical or transcatheter interventions.

How might this impact on clinical practice?

- These data will assist health administrators and clinicians to determine the potential near-term demand for aortic valve interventions and, importantly, whether healthcare services are currently configured to address current to future demands.

Echocardiography Database of Australia (NEDA) patient cohort, the incidence of AS rose eightfold from 5 to 40 cases $/ 1000$ personyears among those aged $<30$ years to $>80$ years (overall incidence of $\sim 18$ cases $/ 1000$ personyears). ${ }^{5}$ Within this same cohort, the proportion of individuals who died with severe AS rose from $3.9 \%$ in those aged $>65$ years to $6.1 \%$ in those aged $>85$ years. ${ }^{6}$ Unfortunately, given the logistics of definitive screening with echocardiography, population studies such as the OxVALVE Study ${ }^{3}$ are scarce. Consequently, the natural history and subsequent disease burden of AS is often described from the perspective of those who are already diagnosed and receiving definitive treatment.

Of concern, in the context of a probable 'iceberg' of largely undetected and untreated cases of AS within progressively ageing highincome countries like the $\mathrm{UK},{ }^{7}$ is the high mortality associated with untreated severe AS, especially once symptoms develop. ${ }^{8}$ The mortality in such patients is reported to be as high as $50 \%$ at 2 years and $97 \%$ at 5 years. ${ }^{9}$ Poor survival rates associated with the full 
spectrum of AS within the large, unselected NEDA cohort ${ }^{6}$ reaffirmed the potential to proactively identify and treat more individuals with severe AS. ${ }^{10}$ In common with the evolving trial evidence around aortic valve replacement (AVR), ${ }^{2}$ within the same cohort, the survival benefits conferred by successful AVR were striking. ${ }^{11}$ Such data highlight the potential for substantial public health benefits of earlier detection of AS, using the stethoscope and echocardiography, and subsequent rapid access to heart valve centres/clinics. ${ }^{2}$ This latent potential to increase the detection of AS was further highlighted in the NHS England's Long-Term Plan 2019. ${ }^{12}$ However, until more definitive population studies of AS are conducted, it is difficult to accurately assess the size of the treatable population with severe AS and thereby plan clinical services to ensure potentially life-saving treatments are applied. ${ }^{13} 14$

\section{STUDY AIMS}

The primary aim of this study was to determine how many people might be expected to need evidence-based treatment for severe AS in the UK in the near term based on expert guidelines for its optimal diagnosis and management. ${ }^{2}$ Specifically, we first sought to apply age-specific estimates of the prevalence of severe AS within the known UK population aged 55 years or older to calculate the likely number of individuals affected by severe AS overall (prevalent cases). We further sought to estimate how many individuals could be immediately identifiable/ detected based on the presence of concurrent symptoms (diagnosed/treated cases). Finally, based on the historical application of surgical aortic valve replacement (SAVR) versus transcatheter aortic valve implantation (TAVI) for severe AS according to surgical risk (based on composite data derived from the UK, Europe and North America), ${ }^{15} 16$ we further aimed to quantify the likely demand for surgical services if all cases of severe AS were detected (surgical caseload).

\section{METHODS}

A pragmatic approach to estimate the treatable burden of disease imposed by severe AS in the UK was applied using similar methods previously used to derive what proved to be an accurate forecast of the contemporary and projected burden of heart failure in the UK. ${ }^{17}$ The same broad methods were also applied to an equivalent study of the treatable burden of AS in Australia. ${ }^{7}$ The primary basis for this study (in respect to providing AS-specific data) was two meta-analyses of AS that systematically reviewed and analysed the relevant public health to clinical literature to build contemporary models of the likely burden and treatment of severe AS. ${ }^{15} 16$ The most recent of these reports synthesised data from 9723 cases of severe AS from 7 studies (predominantly from North America and Europe/UK) ${ }^{16}$ A decision-making flowchart of severe AS (from the prevalent population to how they are then sequentially treated according to their probable symptomatic profile and surgical risk status) was then constructed. The sequential distribution of cases through the flowchart was informed by meta-analyses with beta distributions used at each step (using 10000 Monte Carlo simulations) to derive the most accurate point estimates (with 95\% confidence intervals, CIs) for each sequence. Critically, local reimbursement policies were not considered in determining who might or might not be treated with surgical intervention. ${ }^{16}$ Notwithstanding the rapidly evolving literature around the choice between SAVR versus TAVI and, indeed, the pattern of surgical risk that will undoubtedly influence future treatment pathways, ${ }^{2}$ this model provided a robust framework for estimating the treatable burden of severe AS in the UK.

\section{Summary model}

Figure 1 summarises the key steps applied to generate the estimates provided in this report with consideration of the UK population structure, the underlying prevalence of severe AS, their symptomatic status and their potential treatment based on their likely surgical risk profilenoting that each identified prevalent case is accounted for in the flowchart. Each of these steps (and their source data) are described below.

\section{UK population data}

To determine how many people within the UK are at risk of developing severe AS (our denominator/at risk population group), we first obtained age-specific and sex-specific population data for the calendar year 2019 from the UK Office of National Statistics (online supplemental figure 1). ${ }^{18}$ In that year, of the approximate 66 million people living in the UK, $30 \%$ were aged $\geq 55$ years (20.1 million people). We also collated these data separately for England, Wales, Scotland and Northern Ireland to generate specific estimates for each geopolitical region.

\section{Age-specific prevalence of severe AS}

As our next step, we applied a point prevalence of 3.5\% for severe AS among individuals in the UK aged $\geq 75$ years. This represents a small increase in the age-specific prevalence rate applied in the original flowchart published by Durko and colleagues ${ }^{16}$ to reflect contemporary reports of an increasing incidence of AS in the UK. ${ }^{19}$ Unlike the original reports, we also focused on the likely burden of severe AS among those aged 55-74 years. To derive valid and accurate prevalence estimates for these age groups, we analysed the pattern of severe AS within the large and unselected NEDA cohort (one of the largest ever studies of all forms of AS to date). ${ }^{56}$ Using this cohort, we first validated an overall prevalence estimate of $3.5 \%$ severe AS among those aged 75 years and above as a conservative starting point for our analyses. We also noted the absence of any major sex-specific differences in this regard (concurring with the UK-based data from the OxVALVE Study $\left.{ }^{3}\right)$. Using this age group ( $\geq 75$ years) as a fixed reference point, we then used the observed age profile of severe AS cases below the age of 75 years within 


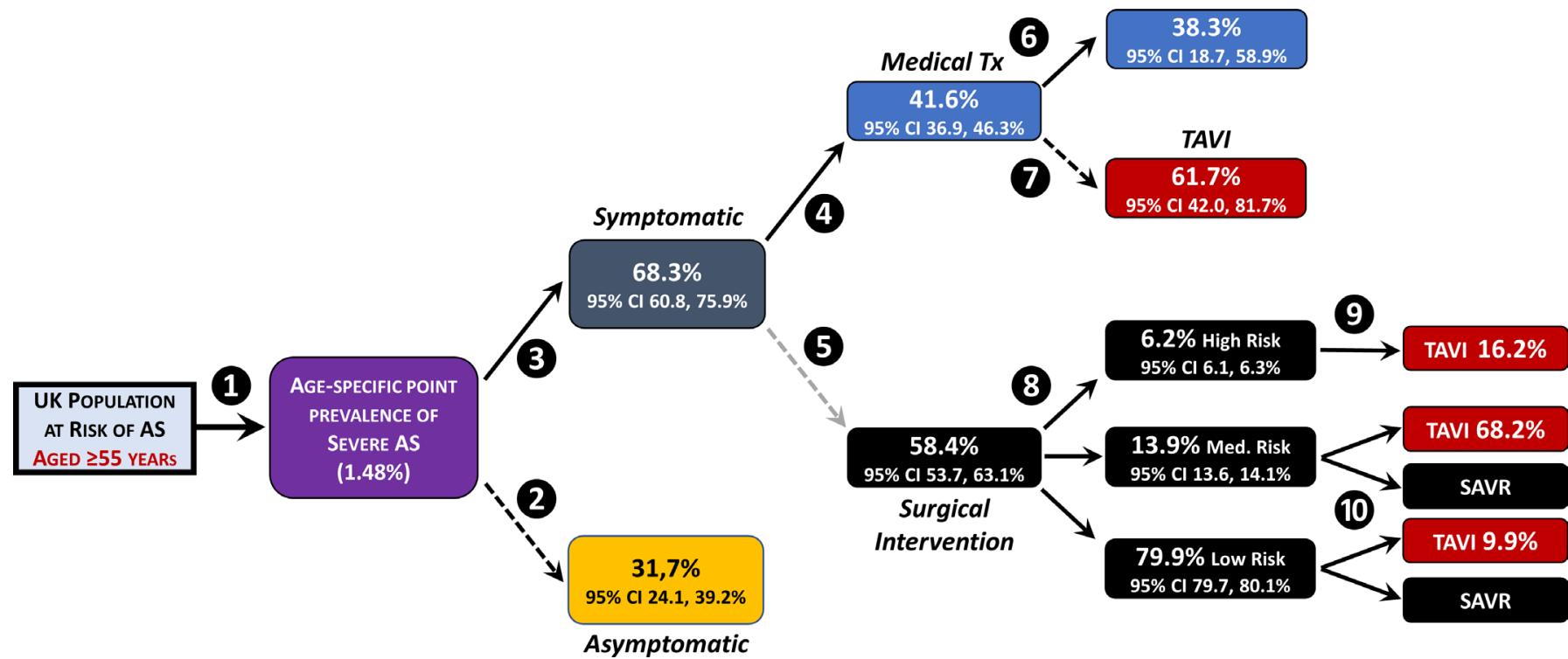

Figure 1 Summary model used to derive treatable burden of disease for severe aortic stenosis (AS) in the UK. To generate our estimates, we first identified the number of individuals aged $\geq 55$ years within the UK population (ie, those most at risk of developing severe AS) and then applied age-specific prevalence rates to this population to determine the total number of cases with severe AS (regardless of their symptomatic or surgical risk status) (1). These cases were then divided into those most likely to be asymptomatic 2 or symptomatic 3. Based on the assumption that symptomatic cases would most likely be detected and, according to current guidelines, ${ }^{2}$ be most likely to be considered for treatment, this 'symptomatic severe AS' group was then divided into those who might be initially considered for medical 4 versus surgical management $\mathbf{5}$. The former was divided into those who would continue to receive conservative medical management $\boldsymbol{6}$ versus those who might benefit from the increasingly accessible option of transcatheter aortic valve implantation (TAVI) $\boldsymbol{\nabla}$. The initially identified surgical cases were then divided according to their surgical risk profile 8 with further stratification of this subgroup according to what proportion of high-risk surgical/surgical aortic valve replacement (SAVR) cases might benefit from TAVI 9 and, similarly, what proportion of low-to-medium risk individuals being considered for SAVR might undergo TAVI instead (10. For each estimate point from 2 to 10 , the equivalent proportion (and 95\% Cl) applied in the original flowchart was applied. ${ }^{15}$

the NEDA cohort to derive the following age-specific prevalence estimates of severe AS to be applied to the UK population in the following age bands: aged $\geq 75$ years (3.5\%-index prevalence estimate), 70-74 years $(1.2 \%)$, $65-69$ years $(0.7 \%), 60-64$ years $(0.5 \%)$ and $55-59$ years $(0.4 \%)$. This age-specific approach differs from the original reports where population estimates (European and North America) were also used as a starting point, but a single, rather than age-stratified, point-prevalence estimate for severe AS was applied. Critically, when these age-stratified estimates were applied to the UK population aged $\geq 55$ years, they generated an overall point prevalence of severe AS that was congruent to that of the estimated prevalence of undetected AS cases within the OxVALVE Study cohort. ${ }^{3}$

\section{Treatable cases}

Having estimated the total number of individuals aged $\geq 55$ years living in the UK with severe AS, we applied the same proportions and 95\% CI developed (derived from meta-analyses of the published literature) and then applied by Durko and colleagues ${ }^{16}$ to determine what proportion of prevalent cases would be symptomatic versus non-symptomatic (this latter group being less likely to be detected and treated) (see figure 1). Of those considered to be symptomatic, we further stratified such individuals (once again using the original proportions and $95 \%$ CIs for these estimates ${ }^{16}$ ) according to their likely suitability for more conservative medical versus surgical treatment and the subsequent option of SAVR versus TAVI (see also figure 1). Specifically, as per the original flowchart/modelling, we distributed SAVR and TAVI treatment according to the likely perioperative risk profile of potentially treated cases according to the Society of Thoracic Surgeon's Predicted Risk of Mortality Score (STS-PROM) ${ }^{20}$ In the absence of more specific UK data, ${ }^{21}$ it is explicitly acknowledged that the distribution of cases according to their surgical risk and options for SAVR versus TAVI will potentially underestimate or overestimate the risk profile of cases and the number of procedures that might be applied both now and the immediate future.

\section{5-year mortality}

To provide an indicative estimation of the potential number of deaths linked to severe AS without proactive treatment in the UK based on the estimated total number of cases living with the condition in 2019, we applied actual 5-year mortality rates observed within the same age groups of the NEDA cohort who did not undergo AVR. Specifically, we assumed during the period 2019-2024, $17.0 \%, 37.5 \%, 43.7 \%, 49.1 \%, 58.6 \%, 69.2 \%$ and $83.7 \%$ of those individuals aged 55-59, 60-64, 65-69, 70-74, 


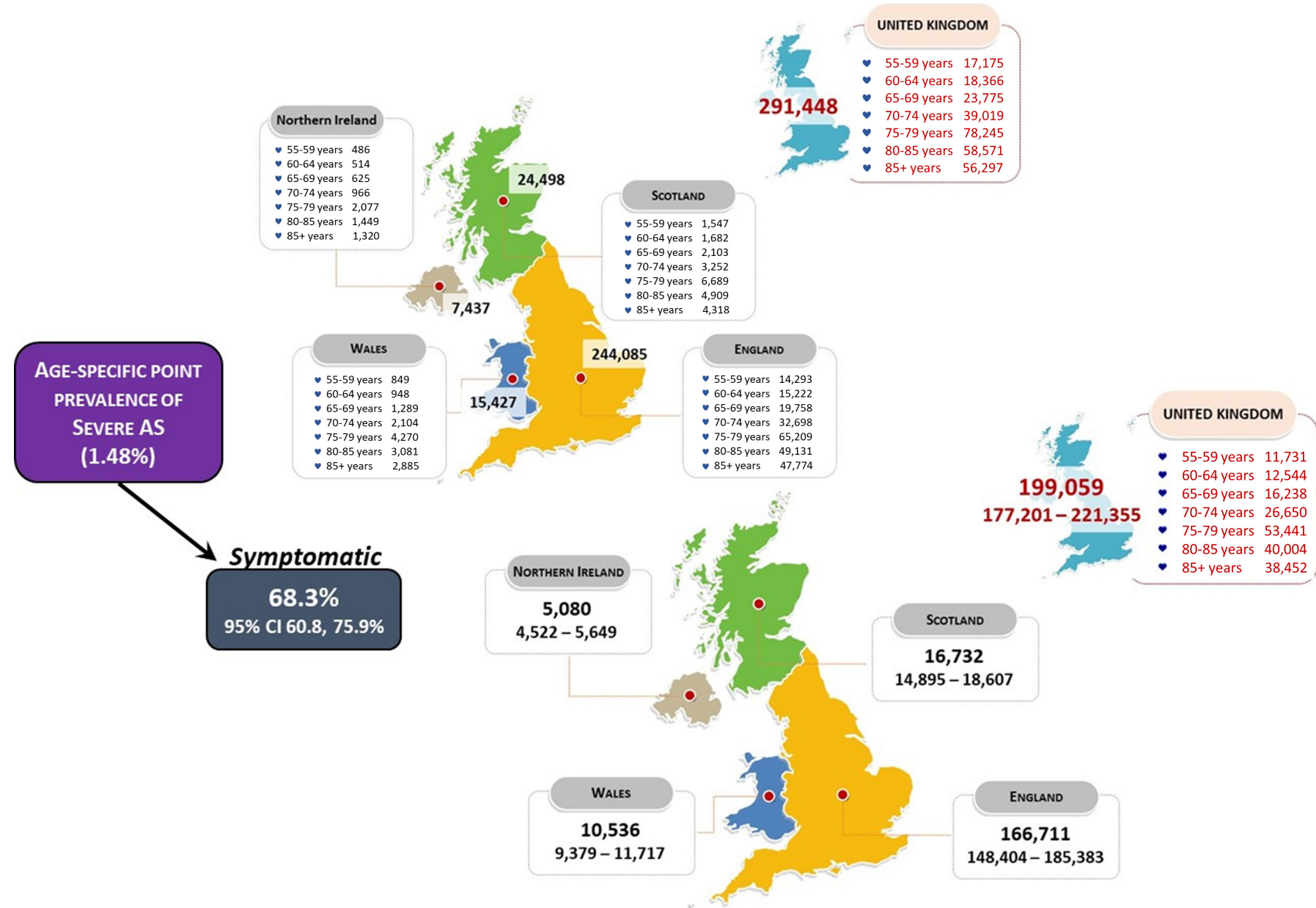

Figure 2 Estimated point prevalence of severe (symptomatic) aortic stenosis (AS) in the UK (2019). This figure shows the estimated prevalence of severe AS across the UK in those aged $\geq 55$ years when applying age-specific prevalence rates (top panel-overall prevalence of $1.48 \%$ as per purple box) in addition to those with symptoms (symptomatic rate with $95 \% \mathrm{Cl}$ shown in dark blue box-bottom panel). All numbers are subject to rounding from the Office of National Statistics data

$75-79,80-84$ and $\geq 85$ years, respectively, with severe AS (regardless of symptomatic status) would die of any cause.

\section{Statistical analyses}

All data are presented in descriptive form with $95 \% \mathrm{CI}$ provided for the main estimates.

\section{Patient and public involvement}

Given the retrospective nature of study analyses, no participants were asked to advise on interpretation or writing of the manuscript.

\section{RESULTS}

\section{Prevalence of severe AS (2019)}

The overall estimated point prevalence of severe $A S$ within the UK population aged 55 years and above in 2019 is $1.48 \%$. As shown in figure 2 (top panel), this currently equates to around 300000 UK men and women living with this potentially deadly condition at any one time. Of these, we estimate that just less than 200000 people will present with symptomatic, severe AS (figure 2, bottom panel). The remainder of cases who remain asymptomatic (92389 (95\% CI $\sim 70000$ to $\sim 144000$ ) people) are unlikely to be detected/diagnosed unless subject to proactive screening for AS or undergoing investigation for another cardiac condition.

\section{Treatment of severe symptomatic AS (2019)}

Overall, we estimate that 82809 out of 199059 symptomatic individuals (95\% CI 74000 to 92000) initially considered unsuitable for surgery would be treated with medical management. Of these cases, 51093 individuals (95\% CI 35 000 to 68 000) might be eligible for/benefit from TAVI. Among the remainder of symptomatic cases, we estimate that 116251 (95\% CI $~ 107000$ to 126000) individuals could be immediately considered for surgical intervention. As shown in figure 3 (distribution of SAVR procedures) figure 4 (distribution of TAVI procedures), based on the projected surgical risk profile of these 199 059 prevalent cases with symptomatic severe AS and the historical distribution of procedures used to treat them, around 116000 and 51000 SAVR and TAVI procedures respectively, would be therapeutically indicated.

\section{5-year mortality among all prevalent cases}

Depending on the type of treatment applied, it is estimated that up to 172859 of the 291448 prevalent cases 


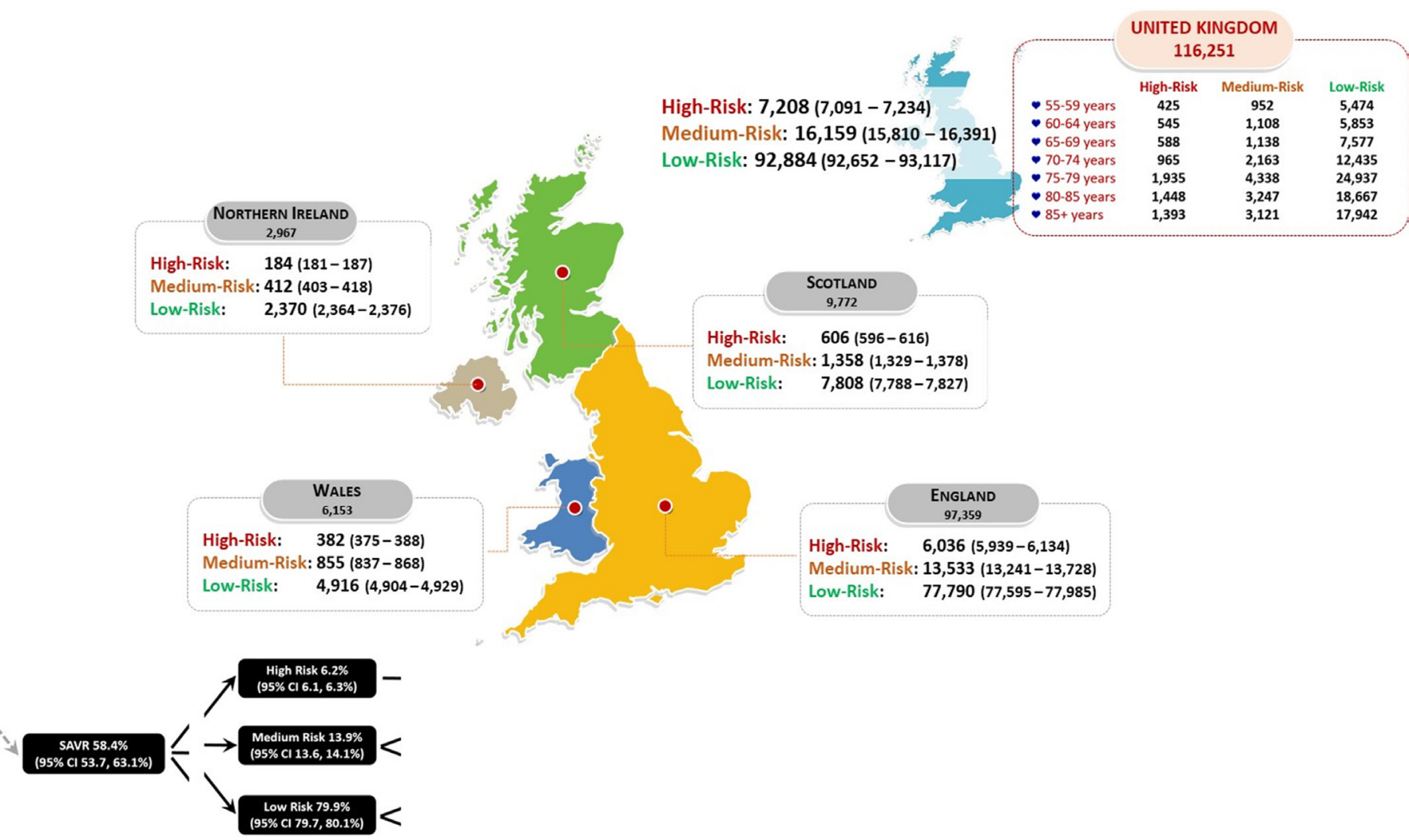

Figure 3 Estimated cases of severe, symptomatic aortic stenosis (AS) in the UK considered for surgical aortic valve replacement (SAVR) (2019). This figure shows the estimated number (with $95 \% \mathrm{Cl}$ ) of prevalent cases of symptomatic, severe AS aged $\geq 55$ years who would be considered for SAVR according to their STS-PROM risk category (rates provided in black boxes).

of severe AS (59.3\% overall) will potentially die during the period 2019-2024 (an annual rate of $\sim 35000$ severe AS-related deaths per annum). Reflective of their lower numbers and more favourable survival rates, we estimate that 9807 deaths $(5.7 \%$ of all deaths) will occur in those initially aged 55-64 years with an additional 29548 deaths (17.1\%) occurring in those aged 65-74 years. Accordingly, most deaths will occur in those aged 75-84 years (86383 deaths-50.0\%) and $\geq 85$ years (47121 deaths$27.3 \%)$.

\section{Overall burden and indicative management of severe AS}

Figure 5 presents the overall, prevalent burden of severe AS in the UK for the year 2019 among those individuals aged $\geq 55$ years. Based on their likely symptomatic/ diagnosed status, it also shows the indicative therapeutic application of AVR according to the strata of estimates described above. In summary, of close to 200000 people aged years and over in the UK estimated to be living with symptomatic, severe AS, around 168000 would potentially benefit from an AVR. Of such individuals, close to 70000 will be aged $<75$ years and therefore at high risk of premature mortality without proactive treatment. Additionally, just over 92000 people will be living with severe AS but without symptoms representing a largely unknown quantity in terms of treatment status.

\section{DISCUSSION}

To our knowledge, this is the first study to specifically estimate the treatable burden of disease associated with severe, symptomatic AS within the UK population. Largely driven by an ageing post-war population cohort, we estimate that close to 300000 adults are currently living with this potentially deadly condition at any one time. Of these, around two-thirds would potentially qualify for SAVR or TAVI based on current guidelines. ${ }^{2}{ }^{22}$ Critically, such an indicative burden is far greater than the current capacity within the NHS to screen, detect, triage and treat such cases. ${ }^{12}$ Moreover, consistent with the prediction of a more than doubling in valvular cases in the UK by 2046 (on the way to a total of 3.3 million prevalent cases in $2056^{3}$ ), based on these prevalence and survival estimates and new data from the NEDA cohort, ${ }^{5}$ we further estimate that the annual number of new cases of severe AS among those aged $\geq 60$ years is close to 29000 . Applying the same surgical risk profile as before, around 20000 people would potentially benefit from SAVR or TAVI.

AS is the most common acquired heart valve disease requiring active clinical intervention. ${ }^{12}$ Its development is typically gradual and can go undetected without active case ascertainment. ${ }^{2324}$ Moreover, the natural history of AS is unpredictable, although the prognosis of severe 


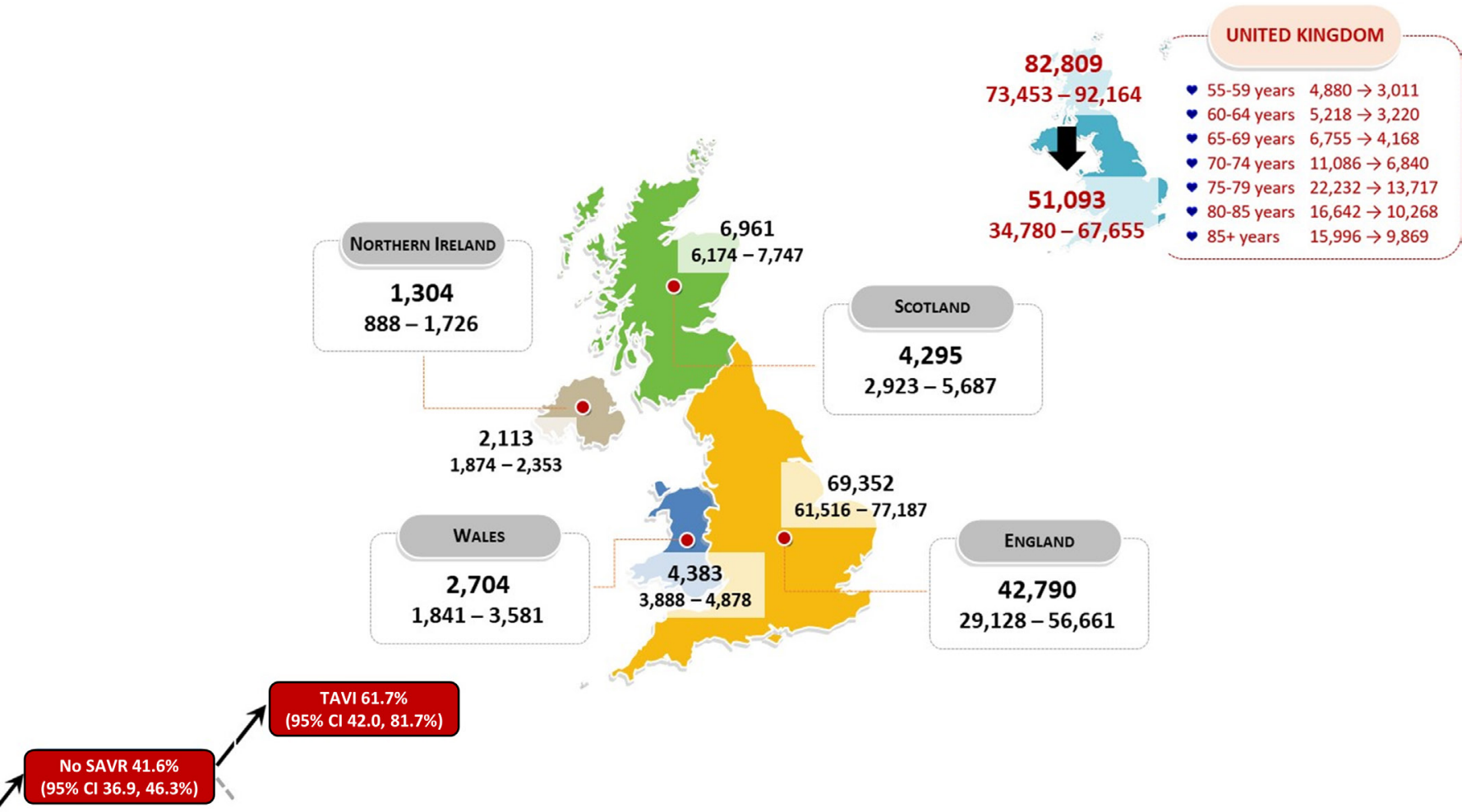

Figure 4 Estimated cases of severe, symptomatic aortic stenosis (AS) in the UK considered for transcatheter aortic valve implantation (TAVI) (2019). This figure shows the estimated number (with $95 \% \mathrm{Cl}$ ) of prevalent cases aged $\geq 55$ years who would be considered too high risk for surgical aortic valve replacement (SAVR) but instead be potential candidates for TAVI (sequential estimate rates for both provided in the red boxes).

AS, especially once associated with symptoms, is often worse than most cancers. ${ }^{1}$ The potential value of early detection of AS and guideline driven interventional treatment in severe AS is substantial, given the evidence that such procedures prolong life. ${ }^{211}$ However, realising this life-saving potential requires an understanding of

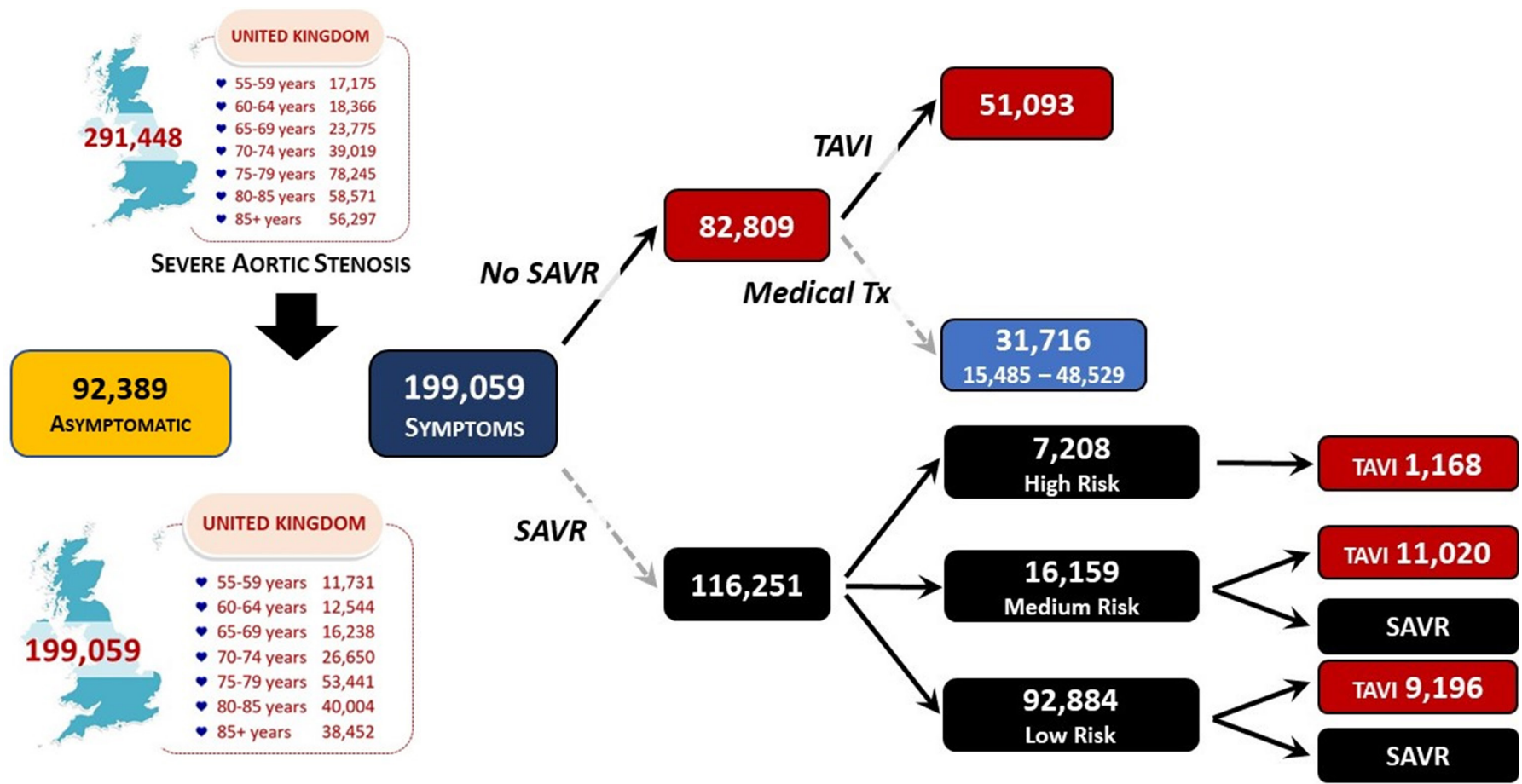

Figure 5 Summary of the estimated (prevalent) burden and management of severe aortic stenosis (AS) in the UK (2019). SAVR, surgical aortic valve replacement; TAVI, transcatheter aortic valve implantation. 
the true size of the population with severe AS who would benefit from treatment and the resources required to meet their therapeutic needs. Building on a previously published model ${ }^{15}$ with specific adjustments derived from $\mathrm{NEDA}^{6}$, we applied a robust set of estimates to determine the treatable burden of severe AS within the UK population. Beyond establishing the existence of a large prevalent population of severe AS (around 300000 people), we further estimate that around 28000 will newly develop this condition each year. To place these figures into a clinical capacity/health service context, in the year 2018-2019 the following procedures were reported to the national audits: 5197 TAVI, 5091 isolated SAVR and 2739 combined SAVR combined with coronary artery bypass grafting (a total of 13027 procedures).$^{25}$ The surgical figures include those from Ireland, and some surgical procedures will have been undertaken for aortic regurgitation rather than AS but making some allowance for this it would be reasonable to suggest that around 10000 procedures were undertaken in the UK that year for severe AS, representing around $50 \%$ of the total we have projected to be developing symptomatic severe AS each year, and therefore being potential candidates for surgical or transcatheter intervention. There seems little doubt therefore that there is a significant shortfall between interventions undertaken for severe AS and our estimates of potential demand. Critically, our estimates of the treatable burden of AS are consistent with the limited data reported previously ${ }^{1516}$ and our overall prevalence estimate of $1.48 \%$ is congruent with the $1.3 \%$ prevalence of undetected AS in the OxVALVE Study. ${ }^{3}$ However, even a small difference between the estimated prevalence ( $1.48 \%$ when combining all-age-specific rates applied) versus actual prevalence of severe AS in the UK will increase the magnitude of error across our projections. Given the substantial cost of treating AS, such uncertainty reinforces the need for contemporary studies of the population and clinical epidemiology of this potentially deadly and disabling condition. ${ }^{2}$

The NHS Long Term Plan (2019) highlighted the need for better detection of heart valve disease and access to specialist care for these patients. ${ }^{12}$ The current study provides estimates for the burden of severe AS, and the likely number of SAVR and TAVI procedures that will be needed to treat this population in an optimal time frame, so that commissioners, and other professionals delivering care, can plan services appropriately. Specifically, the estimated demand will assist the planning of screening processes in primary care (noting the more than 90000 cases estimated to be asymptomatic), and quicker access to secondary care assessment, and receive AVR where appropriate. The requirements for expansion of current facilities, improved training of staff and increased staff numbers will be much easier to estimate based on the modelling produced by this and other research. It will also allow for service improvements that are consistent with national standards and guidelines, such as those soon to be published by the National Institute for Health \& Care Excellence (NICE) and the Getting it Right First Time programme. Recent data from the UK reveals concerning trends with regard to the inequitable access to TAVI, under provision of TAVI for severe symptomatic AS patients, and high rates of mortality for patients on TAVI waiting lists. ${ }^{13}$ Further, recent data from the UK NICOR dataset highlight the detrimental effect of the COVID-19 pandemic on provision of both SAVR and TAVI for existing patients with AS. ${ }^{21}$ Such data highlight the need for a robust and equitable plan to deliver potentially life-saving services to a growing number of people in the UK already affected, or soon to be, by severe AS.

\section{LIMITATIONS}

It is important to note that beyond largely reliable population statistics (the dynamics of which are most important in driving burden of disease estimates), we currently have no means to verify if our overlaying estimates of the prevalence/incidence and treatable burden of disease are correct. Where possible, we have sought to corroborate these vital statistics via the large NEDA cohort. ${ }^{56}$ We also applied the 95\% CI around the key estimates that were synthesised by Durko and colleagues via meta-analyses and Monte Carlo simulations of data from a broad range of countries. However, as noted in the current ESC/EACTS guidelines, STSPROM Scores are inherently variable, and these will influence treatment options. ${ }^{2}$

Notably, beyond a population-based report from the Troms $\varnothing$ Study, ${ }^{26}$ the underlying prevalence and incidence of AS at the whole population level remains poorly characterised. Thus, all our estimates should be cautiously interpreted.

\section{SUMMARY}

In conclusion, this study suggests that severe AS is a common condition affecting many individuals within the UK population aged $\geq 55$ years. Without appropriate detection and intervention, their survival prospects are likely to be poor. However, the indicative treatable burden of disease (around 20000 new, potentially treatable cases each year) is discordant with current capacity within the NHS to deliver AVRs in the form of SAVR or TAVI (regardless of the ratio in which these two different procedures might be applied).

\section{Author affiliations}

${ }^{1}$ School of Medicine, University of Notre Dame, Freemantle, Western Australia, Australia

${ }^{2}$ Department of Cardiology, Royal Prince Alfred Hospital, Sydney, New South Wales, Australia

${ }^{3}$ Centre for Cardiopulmonary Health, Torrens University Australia, Adelaide, South Australia, Australia

${ }^{4}$ School of Medicine, Dentistry and Nursing, University of Glasgow, Glasgow, UK 
${ }^{5}$ Consultant Cardiologist, Faculty of Medicine, University of Southampton \& Wessex Cardiothoracic Unit, University Hospital Southampton NHS Trust, Southampton, UK ${ }^{6}$ Consultant Cardiologist, Manchester University Hospitals Foundation Trust, Manchester, UK

${ }^{7}$ President, Society of Cardiothoracic Surgeons of Great Britain \& Ireland, UK ${ }^{8}$ Consultant Cardiothoracic Surgeon, Sheffield Teaching Hospitals NHS Foundation Trust, Sheffield, UK

${ }^{9}$ Consultant Cardiac Scientist, Manchester University NHS Foundation Trust, Manchester, UK

${ }^{10}$ Consultant Cardiac Surgeon, Royal Infirmary, Edinburgh, UK

${ }^{11}$ Emeritus National Clinical Director for Heart Disease, NHS England, UK

Contributors GS, SS and HHG conceived the study. SS designed and performed the data analysis. GS, SS and HHG wrote the manuscript.GS, SS, NC, SR, SK, PB, $\mathrm{KP}, \mathrm{RP}, \mathrm{DP}$ and HHG interpreted the data and made substantial contributions to the revision and have approved the submitted version. GS accepts full resonsibility for the finished work and/or conduct of the study, had access to the data, and controlled the decision to publish.

Funding The authors have not declared a specific grant for this research from any funding agency in the public, commercial or not-for-profit sectors.

Map disclaimer The inclusion of any map (including the depiction of any boundaries therein), or of any geographic or locational reference, does not imply the expression of any opinion whatsoever on the part of BMJ concerning the legal status of any country, territory, jurisdiction or area or of its authorities. Any such expression remains solely that of the relevant source and is not endorsed by BMJ. Maps are provided without any warranty of any kind, either express or implied.

Competing interests GS: Research grants: Actelion, Bayer, Edwards, GSK, Jensen, Novartis. Consultancies: Edwards, Echo IQ, NEDA Ltd. SS: Research grants: Actelion, Bayer, Edwards, GSK, Jensen, Novartis. NC: Research grants: Boston Scientific, HeartFlow, Beckmann Coulter. Speaker Fees/Consultancy: HeartFlow, Abbott, Edwards. Travel Sponsorship: HeartFlow, Boston Scientific, Biosensors, Medtronic, Edwards. PB: Honoraria: Edwards Lifesciences and Atricure Ltd. DP: Research grants: Actelion, Bayer, Edwards, GSK, Jensen, Novartis. HHG: Consultancies: Wilmington Healthcare, Edwards Lifesciences, Heart Valve Voice.

Patient consent for publication Not applicable.

Provenance and peer review Not commissioned; externally peer reviewed.

Data availability statement All data relevant to the study are included in the article or uploaded as supplementary information.

Open access This is an open access article distributed in accordance with the Creative Commons Attribution Non Commercial (CC BY-NC 4.0) license, which permits others to distribute, remix, adapt, build upon this work non-commercially, and license their derivative works on different terms, provided the original work is properly cited, appropriate credit is given, any changes made indicated, and the use is non-commercial. See: http://creativecommons.org/licenses/by-nc/4.0/.

\section{ORCID iDs}

Geoffrey A Strange http://orcid.org/0000-0001-6800-7119

Simon Stewart http://orcid.org/0000-0001-9032-8998

Nick Curzen http://orcid.org/0000-0001-9651-7829

Simon Ray http://orcid.org/0000-0001-7775-1900

David Playford http://orcid.org/0000-0003-4492-1103

\section{REFERENCES}

1 Nkomo VT, Gardin JM, Skelton TN, et al. Burden of valvular heart diseases: a population-based study. Lancet 2006;368:1005-11.

2 Beyersdorf F, Praz F, Milojevic M. 2021 ESC/EACTS guidelines for the management of valvular heart disease. Eur Heart J 2021;00:1-72.

3 d'Arcy JL, Coffey S, Loudon MA, et al. Large-scale community echocardiographic screening reveals a major burden of undiagnosed valvular heart disease in older people: the OxVALVE population cohort study. Eur Heart J 2016;37:3515-22.
4 Nchimi A, Dibato JE, Davin L, et al. Predicting disease progression and mortality in aortic stenosis: a systematic review of imaging biomarkers and meta-analysis. Front. Cardiovasc. Med. 2018;5:112.

5 Stewart S, Chan Y-K, Playford D, et al. Incident aortic stenosis in 49449 men and 42229 women investigated with routine echocardiography. Heart 2021;8:heartjnl-2021-319697.

6 Strange G, Stewart S, Celermajer D, et al. Poor long-term survival in patients with moderate aortic stenosis. J Am Coll Cardiol 2019;74:1851-63.

7 Strange G, Scalia GM, Playford D, et al. Uncovering the treatable burden of severe aortic stenosis in Australia: current and future projections within an ageing population. BMC Health Serv Res 2021;21:790.

8 Frey N, Steeds RP, Rudolph TK, et al. Symptoms, disease severity and treatment of adults with a new diagnosis of severe aortic stenosis. Heart 2019;105:1709-16.

9 Lancellotti P, Magne J, Dulgheru R, et al. Outcomes of patients with asymptomatic aortic stenosis followed up in heart valve clinics. JAMA Cardiol 2018;3:1060-8.

10 San Román JA, Vilacosta I, Antunes MJ, et al. The 'wait for symptoms' strategy in asymptomatic severe aortic stenosis. Heart 2020;106:1792-7.

11 Playford D, Stewart S, Celermajer D, et al. Poor survival with impaired valvular hemodynamics after aortic valve replacement: the National echo database Australia study. J Am Soc Echocardiogr 2020;33:1077-86.

12 National Health Service, 2021. Available: https://www.longtermplan. nhs.uk [Accessed May 2021]

13 Ali N, Faour A, Rawlins J, et al. 'Valve for life': tackling the deficit in transcatheter treatment of heart valve disease in the UK. Open Heart 2021;8.

14 Chambers JB. Aortic stenosis: service delivery before guidelines. Heart 2019;105:1686-7.

15 Osnabrugge RLJ, Mylotte D, Head SJ, et al. Aortic stenosis in the elderly: disease prevalence and number of candidates for transcatheter aortic valve replacement: a meta-analysis and modeling study. J Am Coll Cardiol 2013;62:1002-12.

16 Durko AP, Osnabrugge RL, Van Mieghem NM, et al. Annual number of candidates for transcatheter aortic valve implantation per country: current estimates and future projections. Eur Heart $J$ 2018;39:2635-42.

17 Stewart S, Maclntyre K, Capewell S. Heart failure and the aging population: an increasing burden in the 21st century? Heart 2003;89:49-53

18 Population estimates for the UK, England and Wales, Scotland and Northern Ireland - Office for National Statistics (ons.gov.uk).

19 Nazarzadeh M, Pinho-Gomes A-C, Bidel Z, et al. Plasma lipids and risk of aortic valve stenosis: a Mendelian randomization study. Eur Heart J 2020;41:3913-20.

20 Serruys PW, Modolo R, Reardon M, et al. One-Year outcomes of patients with severe aortic stenosis and an STS PROM of less than three percent in the SURTAVI trial. Eurolntervention 2018;14:877-83.

21 Martin GP, Curzen N, Goodwin AT, et al. Indirect impact of the COVID-19 pandemic on activity and outcomes of transcatheter and surgical treatment of aortic stenosis in England. Circulation 2021;14:e010413.

22 Otto CM, Baumgartner H. Updated 2017 European and American guidelines for prosthesis type and implantation mode in severe aortic stenosis. Heart 2018;104:710-3.

23 Rosenhek Ret al. Mild and moderate aortic stenosis natural history and risk stratification by echocardiography. Eur Heart $J$ 2004;25:199-205.

24 Delesalle G, Bohbot Y, Rusinaru D, et al. Characteristics and prognosis of patients with moderate aortic stenosis and preserved left ventricular ejection fraction. J Am Heart Assoc 2019;8:e011036.

25 Adult Cardiac Surgery (Surgery Audit). National Institute for cardiovascular outcomes research (NICOR). Available: https:// www.nicor.org.uk/national-cardiac-audit-programme/adult-cardiacsurgery-surgery-audit/ [Accessed Jun 2021].

26 Eveborn GW, Schirmer H, Heggelund G, et al. Incidence of aortic stenosis in subjects with normal and slightly elevated aortic gradients and flow. Heart 2015;101:1895-900. 